\title{
Digital filtering and signal processing
}

\author{
JOHN D. LOVELL*, DAVID C. NAGEL $\dagger$ \\ and EDWARD C.CARTERETTE \\ Perceptual Systems Laboratory \\ University of California, Los Angeles \\ Los Angeles, California 90024
}

The design, realization, and some of the many uses of digital filters are reviewed. Computer programs coded in FORTRAN are presented for the design of lumped parameter digital and analog filter systems which are simulated on a digital computer. Explanations of the programs are accompanied by examples of their use. There is a discussion of problems arising from errors of roundoff and approximation.

Digital filtering is a general mathematical technique of processing sequences of sampled data points which is analogous to the action of electrical or mechanical filtering. Since electrical and mechanical systems may be described in the same way mathematically, the technique is the same in both cases. Only lumped parameter systems are considered. This implies that the systems can be described by ordinary differential equations rather than partial differential equations.

The following discussion often refers to filtering as taking place in the time dimension, but it could equally well be taking place in some other dimension, for example, in space. Thus, reference to a point in time could be as well to a point in any other appropriate dimension.

An example of filtering in the time domain is the telephone, in which frequency and phase are selectively altered by mechanical and electrical filtering. An optical lens does filtering in the spatial domain, and the quality of a lens is often measured by its ability to resolve the pattern of a sine-wave grating.

Digital filtering is a versatile technique for use in simulation, signal processing, and analysis. The techniques can be used with stored data or with data in real time. As computers become faster and memory becomes cheaper, digital filtering will become increasingly useful with both kinds of data. There are many possible applications of digital filtering, as the following examples show.

\section{SIMULATION}

A mechanical or electrical system which is described by a complex equation can be simulated with a digital

\footnotetext{
*Present address: Department of Psychology, California State University, Hayward, California 94542.

tPresent address: Biotechnology Division, NASA AMES Research Center, Moffett Field, California 94035.
}

filter. The Flanagan (1972) basilar membrane model, the Zwislocki (1959) middle ear model, and the linear part of the Lovell, Carterette, and Nagel (1972) basilar membrane model are such systems. In the two basilar membrane models, a point on the membrane is represented as a resonant digital system. The final output of these models is the position of the membrane in time as a function of arbitrary input waveforms.

\section{SIGNAL PROCESSING}

A digital filter can be used in the same way as an analog filter in shaping spectra. In the case of low frequencies, digital filters have a particular advantage over analog filters, since the lower the frequency range of interest, the more easily digital filtering can be carried out in real time. As operating frequency is lowered, analog filters become more expensive, since physically large components are required. An analog waveform can be converted to digital form, processed, and reconverted to analog form in real time, depending on the complexity of the filtering algorithm, the upper range of the frequencies of interest, and the speed of the computer. Components of speech can be synthesized by imitating the filtering action of the vocal system (Schafer, 1972).

\section{SIGNAL ANALYSIS}

Digital filters can be used in the same manner as a waveform analyzer to measure the spectrum of a signal (Weaver, Mantey, Lawrence, \& Cole, 1966). Because the input time sequence could be any sort of sequential data, such as hourly temperature, daily gross receipts, number of social interactions, etc., digital filtering may be useful in analyzing various sorts of business and social science data.

The digital filtering algorithms to be described represent just one of several possible techniques that may be used to process sampled data. Algorithms which are based on linear difference equations will be developed. These methods are termed recursive because they involve sequential operations on past and present inputs and outputs to the filter.

At least two other methods may be used in similar fashion to linear difference equations. In one method, the input time series is convolved with the impulse response of the filter to obtain the filtered output time series. The other method, based on the discrete Fourier transform, is similar except that the convolution is done in the frequency domain. Fourier methods are generally used on subsets of the sample points and thus do not so easily yield running or instantaneous analyses. 
Since, to a great extent, all three techniques may be used to do the same thing, the question naturally arises as to why one method is preferred to another. The answer is that the linear recursive techniques are generally simpler to implement, require less storage and computation time, and are more intuitively related to analog or continuous filter networks through transform theory. In fact, the design of recursive digital filters is simpler than the corresponding design of analog filter networks, because one needn't worry about impedance between adjacent filter sections.

In the following, we shall consider the mathematical background of digital filtering, the design of digital and analog filters, the implementation of digital filters, and the use of the accompanying computer programs. The programs are subroutines for which calling programs must be supplied by the user. Sample main programs are also given to illustrate the use of the subroutines. The techniques presented here should be readily usable in a wide variety of applications. The subroutines are written quite generally. The user may wish to write more specific programs for his particular system so as to save time and memory usage. The programs were coded in FORTRAN IV, but attempts were made to keep the language compatible with FORTRAN II.

\section{MATHEMATICAL BACKGROUND}

A linear recursive digital filter may be thought of as a sampled data system whose output at nT (time, space, etc.) is the weighted sum of:

\section{(1) the input at $\mathrm{nT}$}

(2) previous inputs at $(\mathrm{n}-1) \mathrm{T},(\mathrm{n}-2) \mathrm{T}, \cdots$

(3) previous outputs at $(n-1) T,(n-2) T, \cdots$

where $\mathrm{n}=0,1,2, \cdots, \mathrm{k}, \cdots$ and $\mathrm{T}$ is the sampling interval.

A physical or mathematical system is linear if it possesses two properties: (1) additivity, expressed mathematically as

$$
\mathrm{H}\left(\mathrm{x}_{1}+\mathrm{x}_{2}\right)=\mathrm{Hx}_{1}+\mathrm{Hx}_{2}
$$

and (2) homogeneity, expressed as

$$
\mathrm{H}\left(\mathrm{kx}_{1}\right)=\mathrm{kHx}_{1}
$$

$\mathbf{H}$ is the system operator, a mathematical expression describing how the system operates on inputs; $k$ is a constant, and $x_{1}$ and $x_{2}$ are time-varying input signals to the system which may be periodic, aperiodic, or random. In terms of the spectral representation of signals, these properties imply that no frequency component may appear in the output of the system that did not appear in the input. Examples of non-linear systems that do not meet the above conditions are squaring devices (such as an rms voltmeter) and rectifiers.
Provided that a system meets the linearity conditions noted above, it can be completely described mathematically by an expression termed the system impulse response, which is merely the response of the system to an input signal that is infinitesimally brief.

An intuitive feeling for the meaning of the impulse response may be gained by noting that an ideal impulse is a signal which contains equal power at all frequencies. Thus, the impulse response of a linear system is a signal containing all frequencies. However, the power at a particular frequency is modified by the transmission characteristics of the system. The impulse response thus represents the response of the system to all possible frequencies. Knowing the impulse response, and the fact that a given system is linear, allows prediction of the response of the system to any arbitrary input signal.

Of central importance to a description of linear systems is a technique known as transform theory, or more broadly, operational mathematics. The use of transform methods allows complex mathematical operations (such as integration or differentiation) to be reduced to what are largely algebraic manipulations. This is done by a change of variables in the system-describing equations. If Laplace or z-transform techniques are used (as in the present paper), the time variable, $t$, becomes transformed into a complex variable, $s$. The rules used to perform such transformations are straightforward (Churchill, 1958; Jury, 1964).

Transform techniques provide a convenient connection between the time and frequency domains. This is important since, for the most part, filter specifications are given in terms of frequency and phase response rather than time response (i.e., the impulse response specification). Transforming the equations which describe a system into functions of the complex variable, $s$, instead of a time variable, $t$, allows the correspondence between the time and frequency domains to be made and thus facilitates filter design. Another way of saying the same thing is to note that although filters are designed from considerations of their frequency and phase response, they are ordinarily used to operate on a time series or the sampled equivalent. Thus, the frequency and time correspondence is a necessary and important one.

Continuous time functions such as voltage in a circuit are represented by differential equations. These are functions of a time variable, $t$. The Laplace transform of these equations is a function of the complex variable, $s$, and defines the frequency response of the circuit.

Sampled data systems which are analogous to the continuous time function are represented by difference equations. They are functions of a sampled time variable, nT. The $z$ transforms of such difference equations are analogous to the Laplace transforms of the differential equations.

As previously noted, the impulse response of a linear system completely characterizes that system; that is, we can predict what the output of the system will be for any arbitrary input. Such an impulse response is clearly a 
function of time, $t$. If we transform the impulse expression as a function of the variable, $s$, via a Laplace transformation operation, we have gained the ability to express system performance in the frequency domain. The transformed function is called the system transfer function, usually denoted $\mathrm{H}(\mathrm{s})$. The transfer function, then, is the complex ratio of the output signal to the input signal, where the input signal is an impulse containing all possible frequencies.

In general, the system transfer function is expressed as the ratio of two complex quantities or expressions in the variable, s:

$$
H(s)=\frac{Q(s)}{R(s)}
$$

Although, theoretically, the numerator and denominator may be very complicated, we will consider only some very simple and elementary filter configurations in this paper. As we shall show later, these simple filters may be combined, owing to their linearity properties, into very complicated configurations.

Transfer functions are described in terms of poles and zeroes. As in Eq. 3, the poles of $\mathrm{H}(\mathrm{s})$ are values of $\mathrm{s}$ for which $\mathrm{R}(\mathrm{s})=0$. The zeroes of $\mathrm{H}(\mathrm{s})$ are likewise values of $s$ for which $Q(s)=0$. In other words, poles and zeroes are roots of the functions $\mathrm{R}(\mathrm{s})$ and $\mathrm{Q}(\mathrm{s})$, respectively. All filters to be presented will consist entirely of one of four basic types of poles and zeroes. Single poles and zeroes are those where the roots of $Q(s)$ and $R(s)$ are real numbers. Conjugate pole and zero pairs are those where the roots of $Q(s)$ and $R(s)$ are complex, that is, they have both a real and an imaginary component. The transfer functions of these elementary pole or zero forms are shown in Table 1. The amplitude and phase response are found from the elementary transfer functions by a substitution of the complex angular frequency, $j \omega$, for the complex variable, s. Taking the example of a simple pole:

$$
H(s)=\frac{1}{s+\alpha}
$$

the amplitude response, written $|\mathrm{H}(\mathrm{j} \omega)|$, is obtained through the following steps:

$$
\begin{aligned}
& H(j \omega)=\frac{1}{j \omega+\alpha} \\
& H(j \omega)=\frac{1}{j \omega+\alpha} \cdot \frac{j \omega-\alpha}{j \omega-\alpha}=\frac{j \omega-\alpha}{-\omega^{2}-\alpha^{2}}=\frac{\alpha-j \omega}{\alpha^{2}+\omega^{2}}
\end{aligned}
$$

Then, define

$$
\operatorname{Re}=\frac{\alpha}{\alpha^{2}+\omega^{2}}
$$

$$
\begin{gathered}
\operatorname{Im}=\frac{-\omega}{\alpha^{2}+\omega^{2}} \\
|H(j \omega)|=\sqrt{\operatorname{Re}^{2}+} \frac{\operatorname{Im}^{2}}{\alpha^{2}+\omega^{2}}=\frac{1}{\alpha^{2}}
\end{gathered}
$$

In a similar manner, the phase response may be found as a function of the angular frequency, $\omega$ :

$$
\begin{aligned}
& \varphi(\omega)=\tan ^{-1}\left(\frac{-\mathrm{Im}}{\operatorname{Re}}\right) \\
& \varphi(\omega)=\tan ^{-1}\left(\frac{\omega}{\alpha}\right)
\end{aligned}
$$

\section{DESIGN OF DIGITAL AND ANALOG FILTERS}

Subroutines RESP and RESPS are used together to design digital and analog filters by computing the amplitude and phase response of a linear system transfer function. These routines compute the response for combinations of the transfer functions shown in Table 1 . If equations are constructed by combining these component functions, the effect is to sum the effects of the separate components. The equations are linear. Since digital filters approximate continuous filter systems, we can design an analog filter and then realize the design digitally. There are some inherent dangers in this approach which will be discussed in the section on errors and approximations.

\section{ANALOG FILTER FUNCTIONS}

The amplitude and phase responses of single real axis poles and zeroes are shown in Fig. 1. Poles and zeroes have complementary phase and amplitude response.

As shown in Fig. 1, single poles have an asymptotic amplitude change of $-6 \mathrm{~dB}$ per octave and a total phase shift of $\pi / 2$ at high frequencies. Single zeroes have the same range of phase shift and an asymptotic amplitude change of $+6 \mathrm{~dB}$ per octave. A pole (or zero) at $\pm \alpha$ for $|\alpha|>0$ has a 3-dB less (or greater) response for an input frequency $\omega$ equal to $\alpha$ than for $\omega$ equal to zero. Phase shift at this frequency is half of the total, or $\pi / 4$.

Poles always produce a lagging phase shift, and zeroes always produce a leading phase shift. As shown in Fig. $\mathrm{lb}$, negative poles and positive zeroes (or vice versa) have the same form and direction of phase shift, but differ by $\pi$ from each other.

Conjugate pole or zero pairs at $\pm(\alpha \pm j \beta)$ result in double the asymptotic phase $(\pi)$ and amplitude $(12 \mathrm{~dB}$ per octave) effects of single poles or zeroes. Pole pairs may exhibit a resonant response, as shown in Fig. 2 Zero pairs have a corresponding antiresonant response. The phase response, $\varphi(\omega)$, and amplitude response, $|\mathrm{H}(j \omega)|$, of conjugate pairs are affected by the ratio $\alpha / \beta$.

$$
|H(j \omega)| \rightarrow \infty \text { when } \omega=\beta, \frac{\alpha}{\beta} \rightarrow 0
$$


Table 1

Real Axis Pole

$H_{1}(s)=\frac{1}{s+\alpha}$

$$
G_{1}(s)=\frac{1}{1-e^{-(A+S)}}
$$

Real Axis Zero

$H_{2}(s)=s+\alpha$

$$
G_{2}(s)=1-e^{-(A+S)}
$$

Conjugate Pole Pair

$H_{3}(s)=\frac{1}{(s+\alpha)^{2}+\beta^{2}}$

$$
G_{3}(s)=\frac{1}{1+e^{-2(A+S)}-2 \cos B e^{-(A+S)}}
$$

\section{Conjugate Zero Pair}

$H_{4}(s)=(s+\alpha)^{2}+\beta^{2}$
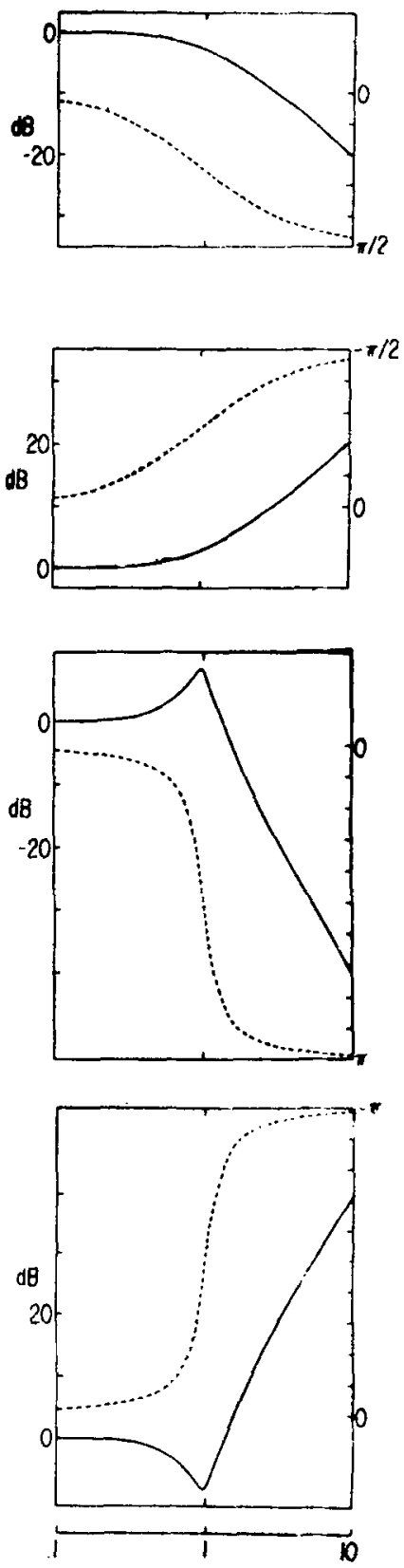

Radian frequency $=$ frequency in cycles per time unit $\times 2 \pi ; \alpha, \beta$, and $\omega$ are radian frequencies.

$T$ is the sampling interval, the inverse of sampling rate; $A, B$, and $W$ are radian frequencies $\times T$.

$s$ is a complex variable; $S=s T, A=\alpha T, B=\beta T, W=\omega T$.

$H(s)$ refers to a component of a continuous filter system.

$G(s)$ refers to a digital filter component which approximates the appropriate $H(s)$.

$$
Z^{-1}=e^{-S}=e^{-s T} \quad H(j \omega)=R e+j I m \quad|H(j \omega)|=\sqrt{\operatorname{Re}^{2}+I^{2}} \quad \varphi(\omega)=\operatorname{Tan}^{-1}(-I m / R e)
$$

$$
\varphi(\omega)=\pi / 2 \text { when } \omega=\sqrt{\beta^{2}+\alpha^{2}}
$$

As $\alpha / \beta$ varies, both the bandwidth and the center frequency of the filter change. Thus, care should be taken not to consider a pole pair as a bandpass filter centered at $\beta$ unless $\alpha / \beta$ is small. The actual oscillatory or peak frequency (the frequency at which response is maximum) is expressed by

$$
f_{o s c}=\sqrt{\beta^{2}-\alpha^{2}}
$$


Figure 3 shows the change in oscillatory frequency and the $3-\mathrm{dB}$ bandwidth of a conjugate pole pair as a function of the $\alpha / \beta$ ratio. The same discussion applies in an inverse way for conjugate zero pairs. If we wish to place a pole pair with a specified oscillatory frequency $X$ and an $\alpha / \beta$ ratio $K, \beta$ should be chosen such that

$$
\beta=\frac{X}{\sqrt{1-K^{2}}}
$$

It is generally not desirable to place poles in the right half of the s plane (i.e., $\alpha>0$ ), because this results in an unstable impulse response which does not damp out, but increases without bound over time. Note that the pole and zero locations can be plotted on a graph whose abscissa is the real axis and whose ordinate is the $\mathbf{j}$ axis. For example, a single pole is located on the abscissa of such a diagram, since it has no imaginary component. If the single pole value is negative, it occurs on the abscissa to the left of the $j$ axis and is said to occur in the left half of the s plane. Such plots graphically represent the associated transfer functions.

If like components are paired on opposite sides of the j axis, the phase effects will be constant over frequency and leave only amplitude effects (for instance real axis zeroes at + and $-\alpha$ ). If poles and zeroes are paired across the $j$ axis, the amplitude effects cancel, leaving only phase effects (for instance, a single pole at $-\alpha$ and a single zero at $+\alpha$ ). This latter combination is known as an all-pass network, since all frequencies are passed with equal amplitude but at different relative phases.

A single pole comprises a low-pass filter. A single zero at 0 and a single pole at a higher frequency make a high-pass filter. A pole pair combined with a zero at the origin can make a bandpass filter with an asymptotic $6 \mathrm{~dB}$ per octave rejection of higher and lower frequencies. This type of bandpass filter is also interesting in that phase is zero at the oscillatory frequency, which is expressed by

$$
f_{\text {osc }}=\sqrt{\beta^{2}+\alpha^{2}}
$$

A bandpass filter made up of two pole pairs at $-\alpha \pm \mathrm{j} \beta$ and a single zero at the origin will tend to keep its maximum amplitude point at $\beta$ as the $\alpha / \beta$ ratio changes. To see this, consider the simultaneous action of Eqs. 14 and 16.

\section{DIGITAL FILTER FUNCTIONS}

The techniques for analysis of continuous and sampled data systems are sufficiently parallel so that, having determined the pole-zero locations necessary to implement an analog filter of the desired configuration, these constants may directly be employed in the sampled data system. The $\mathrm{z}$ transform is the sampled time equivalent of the Laplace transform. In the design of an analog filter, a transfer function was found which described the desired system performance in the
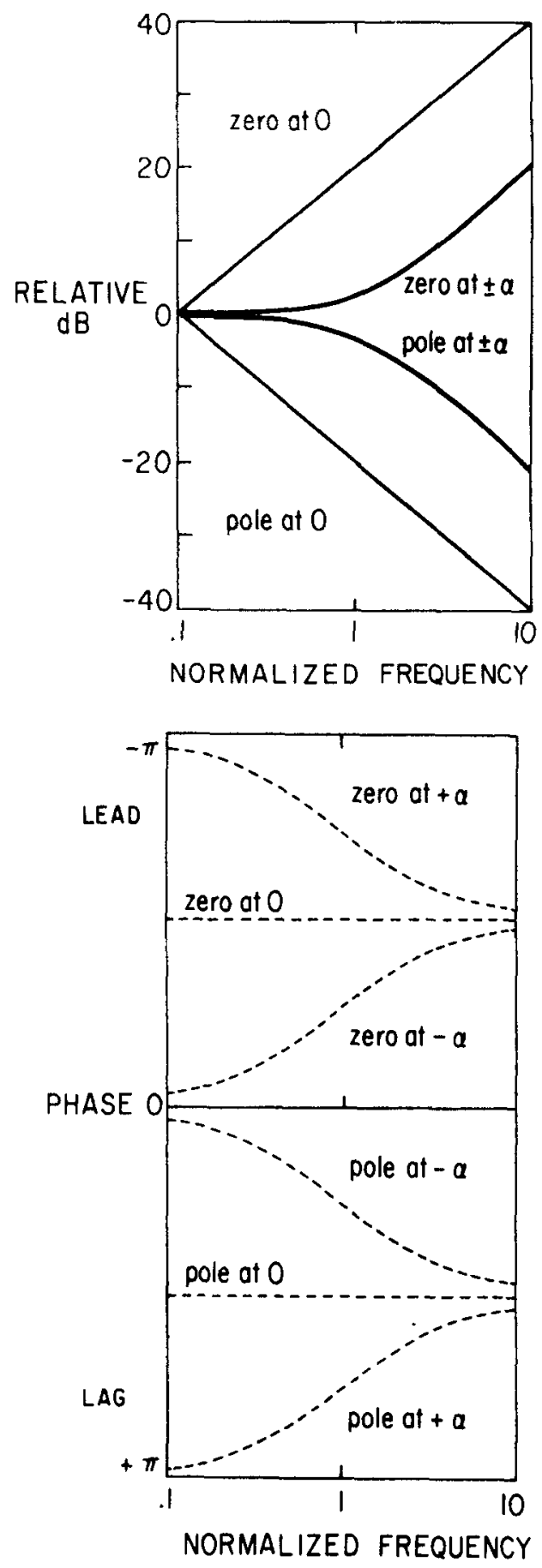

Fig. 1. (a) Relative amplitude effects of real axis poles and zeroes with frequency normalized to $\alpha$. (b) Phase effects of real axis poles and zeroes with frequency normalized to $\alpha$.

frequency domain. In order to derive an equivalent sampled data transfer function, a technique known as the impulse invariant method of digital filter design is used (Gold \& Rader, 1969). In using the method, it is assumed that the impulse response of the digital filter is equal to the sampled impulse, response of a given continuous filter. In practice, it is possible to derive the digital transfer function from the continuous filter transfer function via the following correspondence: 


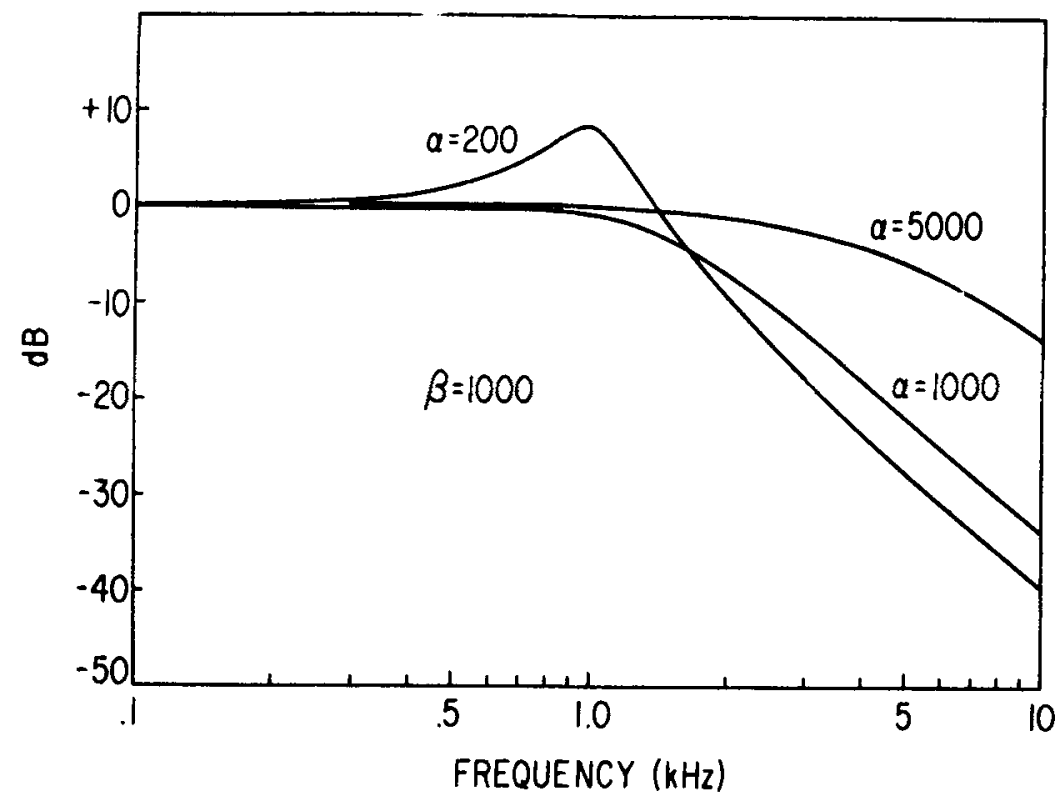

Fig. 2. Conjugate pole pair $\mathrm{H}_{3}$ of Table 1 exhibits a resonant response as $\alpha$ becomes small in comparison with $\beta . \alpha$ and $\beta$ are given in hertz.

to the continuous equivalent, the Laplace transform.

Digital filter functions are difference equations which can be derived from the $G$ functions of Table 1 by inverse $\mathrm{z}$ transformation. The transfer function is equal to the ratio of the output to the input. If $Y(z)$ is the $z$-transformed output sequence and $X(z)$ the z-transformed input sequence, then:

$$
G(z)=\frac{Y(z)}{X(z)}
$$
continuous transfer function on the left. Notice that the same constants appear in both expressions. Having derived the constants for the continuous case, it is only necessary to substitute them into the expression on the right to obtain the sampled data system transfer function. The interested reader should consult Gold and Rader (1969) for a more complete discussion of use of the $\mathrm{z}$ transform in digital filter design and its relationship

Taking the example of a single real axis pole transfer function:

$$
G(z)=\frac{1}{1-e^{-A} z^{-1}}=\frac{Y(z)}{X(z)}
$$

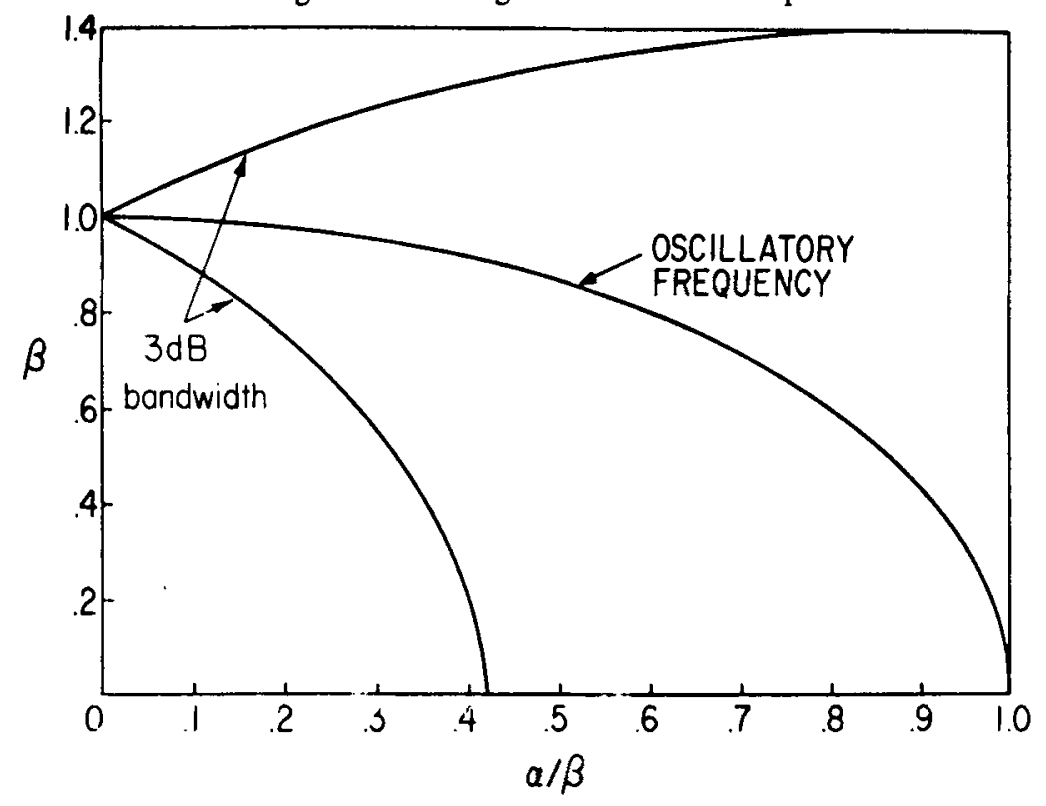

Fig. 3. The oscillatory frequency of a conjugate pole pair (the frequency at which response is maximal) approaches $\beta$ as the $\alpha / \beta$ ratio becomes small. At the same time, the 3-dB bandwidth becomes small. 


$$
\begin{aligned}
& Y(z)=\frac{X(z)}{1-e^{-A} z^{-1}} \\
& Y(z)=Y(z) e^{-A} z^{-1}+X(z)
\end{aligned}
$$

where $\mathrm{A}=\alpha \mathrm{T}$.

Taking the inverse transform ( $\mathrm{e}^{-\mathrm{A}}$ is a constant and $\mathrm{z}^{-1}$ represents a one-sample time delay):

$$
Y(n T)=Y[(n-1) T] e^{-A}+X(n T)
$$

This is the desired first-order linear difference equation. The computational block for this difference equation is found in Fig. 4a. Tables of $z$ transforms are of ten useful in finding inverse transforms for complicated transfer functions (see, for example, Jury, 1964).

Complex or higher order filters may be constructed in two basic ways. For example, a sixth-order resonant filter may be constructed either directly or by cascading three second-order conjugate pole pairs together. By direct construction, we mean finding the roots of a sixth-power polynomial written such that the root or pole locations lead to the desired filter.

The direct construction method is to be preferred in general with analog filters because of interactions between cascade stages. For digital filters, adjacent filter sections are independent. Cascading lower order filters is desirable if the filters are to be implemented on a small computer with a modest word length. This is the case since the coefficients of higher order filter equations must be specified with progressively greater precision in order to maintain their design specifications.

The right half of Table 1 shows the sampled system transfer functions equivalent to the continuous filter transfer functions displayed in the left half of that table. In reference to Table $1, G_{1}$ is the impulse invariant equivalent of $H_{1}, G_{3}$ is not the impulse invariant equivalent of $\mathrm{H}_{3}$, but is very similar and simpler to implement.

The digital filter function, $G(z)$, approximates the continuous filter function, $\mathrm{H}(\mathrm{s})$, when the sampling period, $T$, is small. Figure 4 shows how the digital transfer functions of Table 1 are realized in computational blocks. The $\mathrm{D}$ box is a time delay of one sampling period. The triangular boxes are amplifiers whose gain is given by the value of the constant inside each box. $\mathrm{C}_{0}$ is an inverse gain factor which makes the gain equal to one at a given input frequency, $\omega$.

$$
C_{0}=\frac{1}{|G(j \omega)|}
$$

In Figs. $4 a$ and $4 b$,

$$
C_{1}=e^{-A}
$$

In Figs. $4 c$ and $4 d$,

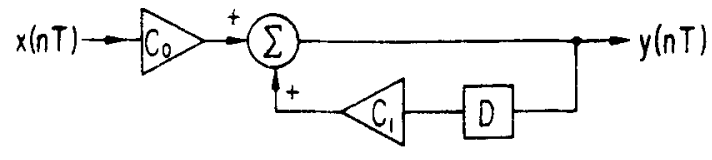

(a)

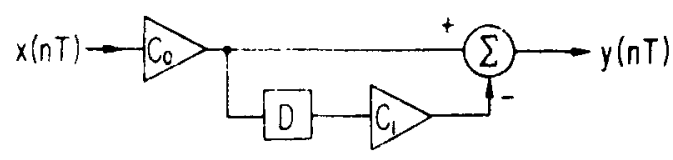

(b.)

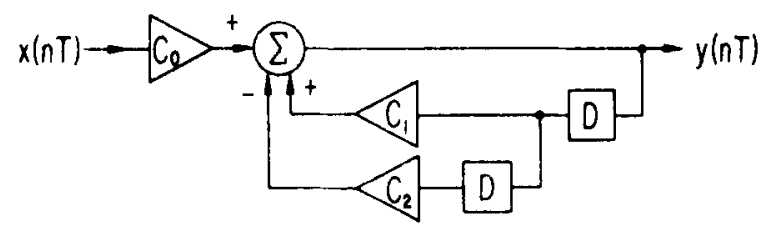

(c)

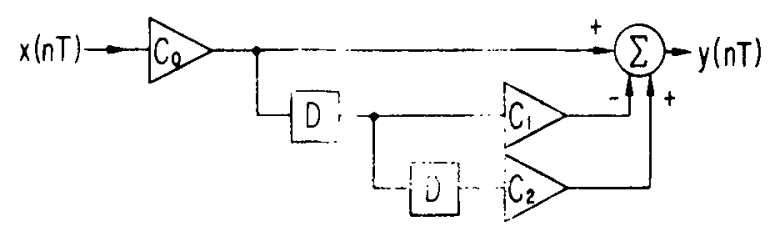

(d)

Fig. 4. Block diagrams of the difference equations associated with the transfer functions $G_{1}, G_{2}, G_{3}$, and $G_{4}$ of Table 1 . These difference equations are the four basic digital filters considered in the paper. $C_{0}$ is a gain factor used to scale the output with respect to the input. See text for explanation.

$$
\begin{aligned}
& C_{1}=2 e^{-A} \cos B \\
& C_{2}=e^{-2 A}
\end{aligned}
$$

where $\mathrm{A}=\alpha \mathrm{T}$ and $\mathrm{B}=\beta \mathrm{T}$.

Figure 5 gives a graphic representation of the difference between $H(s)$ and $G(s)$. Figure $5 a$ is the s-plane representation of a pole pair at $2 \pi$ $(-500 \pm 1000 \mathrm{j})$ and a single zero at $2 \pi(1000)$. Figure $5 b$ represents the sampled data equivalent system for a sampling rate of $5,000 \mathrm{~Hz}$. In the second case, the components repeat infinitely on the $j$ axis. Since the scale of the plane is dependent on $T$, it is apparent that as $\mathrm{T}$ becomes smaller, components move toward integer values on the $\mathrm{j}$ axis.

In general, a digital filter should be used only in the frequency range of from zero $(\mathrm{dc})$ to the Nyquist frequency (half the sampling rate). As shown in Fig. 6, amplitude response reflects around the Nyquist frequency and phase response reflects with opposite sign. At negative frequencies or frequencies higher than the Nyquist frequency, the same pattern repeats. This can lead to aliasing errors if care is not taken to ensure that frequencies higher than the Nyquist frequency are removed from the signal prior to processing. In some 


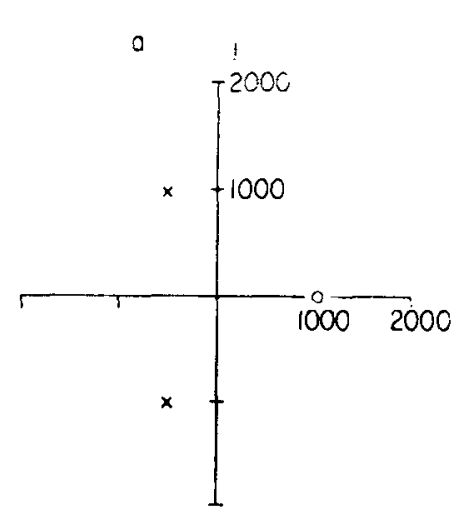

UNITS IN MULTIPLES OF $2 \pi$

sense, it might be said that digital filters do a better job of simulating amplitude response than phase response, since phase response returns to its zero frequency value twice as fast as does the amplitude response. It should be clear from Fig. 6 that in order to closely simulate a given continuous filter system, it is necessary to use a sampling rate considerably higher than twice the highest frequency of interest.

For conjugate pairs, some other effects of interest occur. For conjugate poles, the oscillatory frequency can be more precisely expressed as

$$
f_{o s c}=\cos ^{-1}(\cos B \cdot \cosh A)
$$

Low-frequency phase is distorted as a function of both high gain and low sampling rate, as shown in Fig. 7.

\section{ERRORS OF ROUNDOFF AND APPROXIMATION IN DIGITAL FILTERS}

The programs presented in this paper must be used cautiously and with understanding. A leading authority on digital filtering, Kaiser (1966), says, "... In filter simulation work it is imperative that the user fully
Fig. 5. (a) Representation of a conjugate pole pair and a real axis zero, as in Eqs. $\mathrm{H}_{3}$ and $\mathrm{H}_{2}$ of Table 1 , respectively. (b) Representation of the same conjugate pole pair and real axis zero as Fig. $5 \mathrm{a}$ in a sampled data system with a sampling rate of 5,000 samples/sec, as in Eqs. $G_{3}$ and $G_{2}$ of Table 1, respectively. Components repeat infinitely on the $\mathrm{j}$ axis.

understand the nature of the approximations being made in the design method. The user should also be informed concerning the numerical problems involved in both the digitalization process and the use of the obtained filter [pp. 221-222]."

A computer word has a finite length. Whenever the result of a computation requires storage in excess of the word length, an error arises. The value of an analog signal likewise must be approximated due to sampling errors, quantization errors, and finite word length. Roundoff errors arise from the finite representation of numbers in the machine, and truncation errors arise from the finite representation of processes. Digital filtering is prey to both classes of errors.

In general, the output of a digital filter will be noisy due to errors arising in the approximation of coefficients of parameters, in quantization, and in iteration. Consider as an example the simple first-order filter

$$
y(n T)=K y[(n-1) T]+x(n T) .
$$

It has the solution

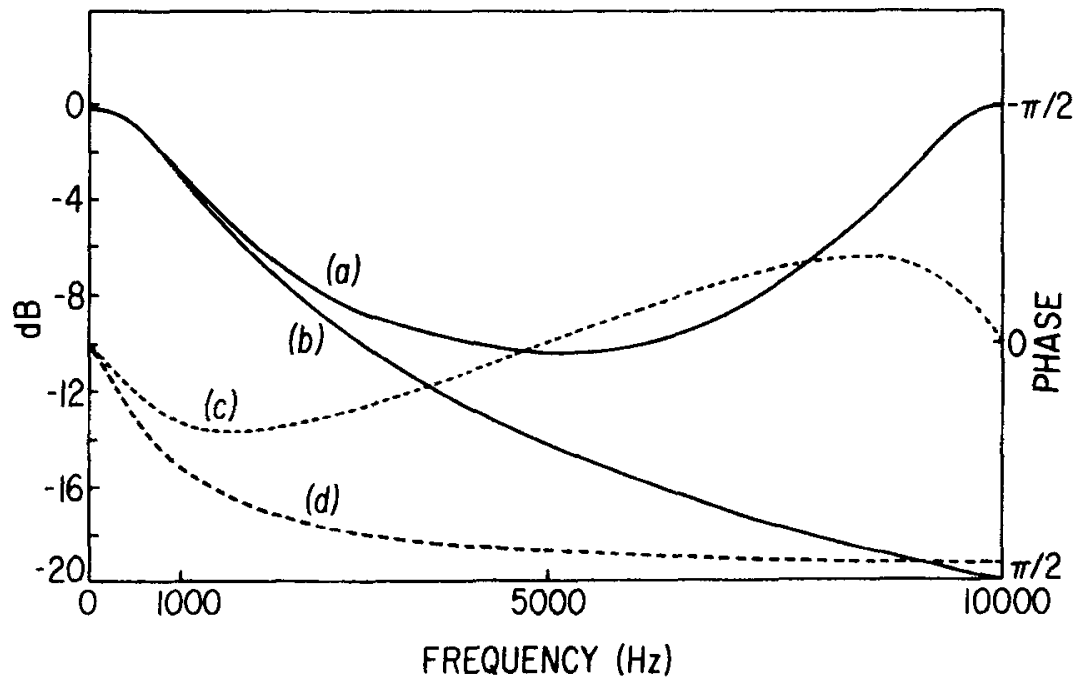

Fig. 6. Comparison of the amplitude and phase effects of an analog real axis pole and the digital equivalent with a sampling rate of 10,000 samples/sec. Solid lines read on the left-hand scale; broken lines read on the right-hand scale. (a) The digital system amplitude function repeats at multiples of the sampling rate. (b) The analog system amplitude function. (c) The digital system phase function repeats at multiples of the sampling rate and goes to zero at the half sampling rate. (d) The analog system phase function. 


$$
y(n T)=K^{n+1} y(-T)+\sum_{i=0}^{n} K^{i} x[(n-i) T]
$$

The coefficient or "gain" parameter, $\mathrm{K}$, is approximated in a register of finite length. The input $x$ suffers from quantization error as well. The iteration of the equation over $\mathrm{n}$ trials involves multiplication and addition roundoff or truncation errors.

When a digital filter is realized in fixed-point arithmetic, round-off or truncation errors of multiplication are the main concern. But when floating-point arithmetic (as in the present paper) is used, roundoff errors occur both in multiplication and addition (see Hamming, 1971).

Beyond this, the error in the output will be different, depending on how the filter is realized mathematically as well as how it is represented in the machine. The same filter may be synthesized in a variety of ways-by direct convolution, by linear recursive equations, or by means of the fast Fourier transform. The amount of error, or noise, in the output depends on the method used. A network of a given order can have several equivalent transfer functions, $\mathrm{H}(\mathrm{s})$. However, each will behave differently in respect to the amount of computational error. This is so because a network can be arranged in cascade or in parallel form, or in mixtures of the two forms.

We have discussed only one method of constructing complex recursive filters from simple first- and second-order elements. It is called the serial, or cascade, method since the output from each simple element is fed serially into the next. However, a given recursive filter can be realized in at least three forms: (1) in cascade, (2) in parallel, or (3) directly as a higher order difference equation. The direct form generally requires greater word length than cascade or parallel forms.

If the length of a computer word is insufficient to represent filter coefficients exactly, errors arise as discrepancies in the amplitude magnitude function of the filter. By setting error bounds on the magnitude function, Avenhus (1972) determined word lengths for the direct, cascade, and parallel forms. For the case of an eighth-degree bandpass filter, Avenhus found that a 14-bit word was sufficient to meet the error bounds on coefficients for either the cascade or parallel forms, whereas a 24-bit word was required for the direct form. Gold and Rader (1969) recommend that all recursive digital filters be put in simple cascade or parallel form.

Inaccuracies in the values of coefficients may be increased by an unduly high sampling rate, a counterintuitive result! However, as the sampling period, $\mathrm{T}$, becomes brief beyond a certain point, the performance of some digital filters deviates further from that of the analog filter (Gold \& Rader, 1969). In particular, larger errors go with low frequencies and high sampling rates because of the stringent need for accurate coefficients.

The amount of noise in the output due to errors in

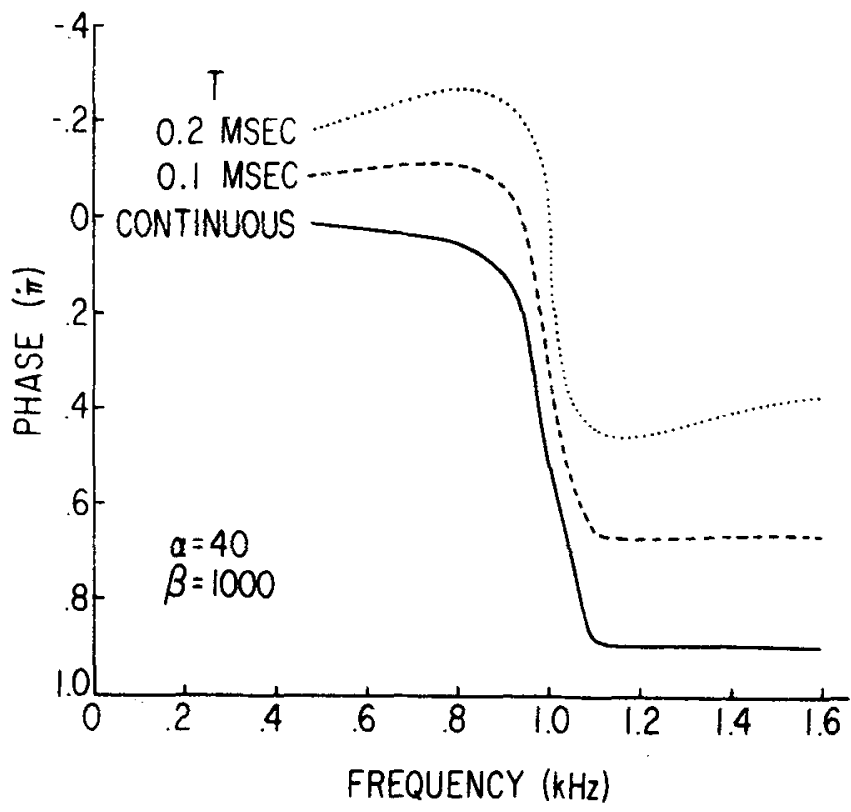

Fig. 7. The phase of a digital conjugate pole pair can shift negative at low sampling rates. $\alpha$ and $\beta$ are given in hertz.

specification or to quantization at the input are relatively easy to estimate. For example, input quantization errors of rounding or truncating may, under certain reasonable assumptions, be treated as noise added to the analog signal source of mean square value $\mathrm{q}^{2} / 12$, where $\mathrm{q}$ is the quantizing step size. More difficult is the case of quantizing error arising from iterative multiplications. As Kaiser (1966) and Gold and Rader (1969) point out, this kind of error depends strongly upon how the quantization is done and upon the specific form of the digital filter.

There have been two main attacks on the problem of errors due to iteration. One way is to compute directly the upper bounds on errors. The other way is to derive statistical measures. The direct method gives upper bounds that are mainly pessimistic, whereas the data agree well with predictions of the statistical models (Oppenheim, 1969).

\section{PROGRAMS FOR DIGITAL AND ANALOG FILTER DESIGN}

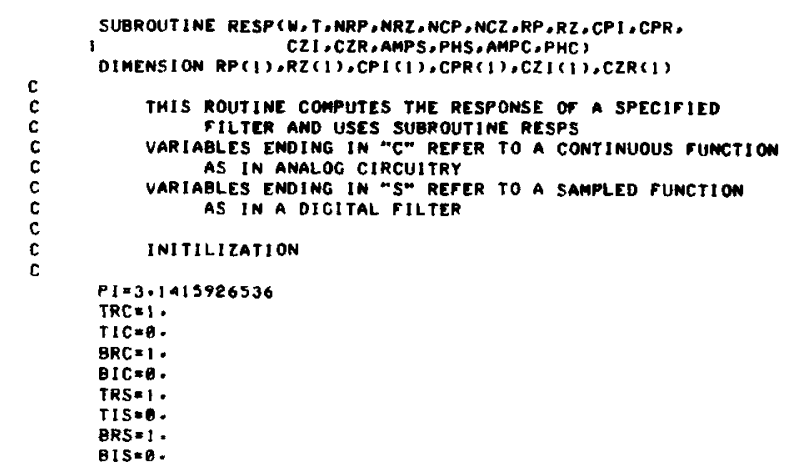



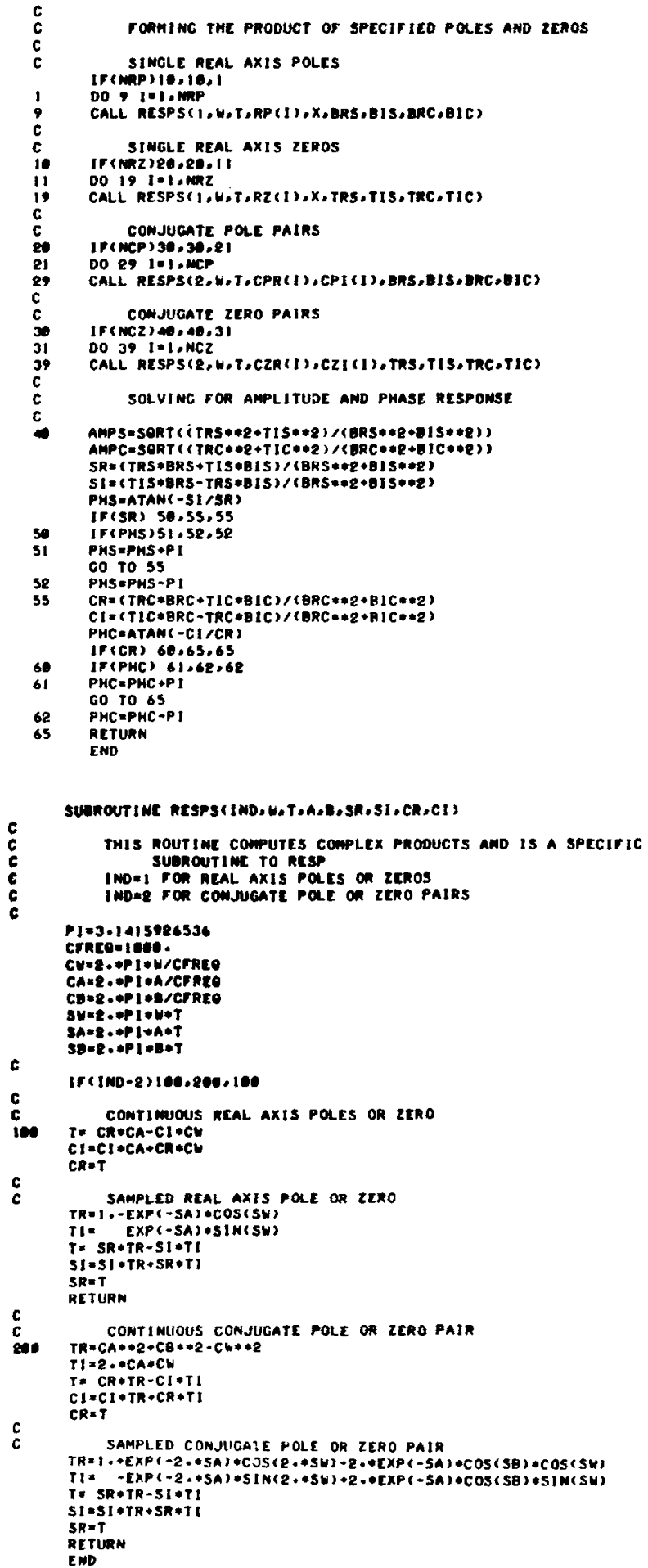

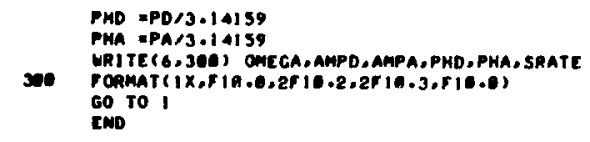

To use the programs RESP and RESPS, a calling program must be written by the user. The main program must get the values which specify a filter and then call RESP. RESPS is called by RESP. Values for the response of the specified filter are retumed to the calling program by RESP. MRESP is a sample main program for the purpose of illustration.

\section{EXPLANATION OF VARIABLE NAMES Variables Passed to RESP}

$W$-the frequency input for which the steady-state filter response is measured.

$T$-the sampling interval, the inverse of sampling rate. NRP-number of real axis poles.

NRZ-number of single zeroes.

NCP-number of complex conjugate pole pairs.

NCZ-number of double zeroes.

$\mathrm{RP}$-an array of single-pole frequencies in hertz [poles are at $-R P(I)]$.

$\mathrm{RZ}$-an array of single-zero frequencies in hertz.

CPI and CPR-arrays of double-pole specifications [poles are at $-\mathrm{CPR}(\mathrm{I}) \pm \mathrm{CPI}(\mathrm{I})]$

CZI and CZR-arrays of double-zero specifications.

\section{Variables Returned by RESP}

AMPS and PHS-the amplitude and phase response of a digital system as specified by the preceding variables. This is the result of combining the transfer functions for sampled data functions [G(s)], as described in Table 1.

AMPC and PHC-these are comparable response variables for the continuous equations $[\mathrm{H}(\mathrm{s})]$, as shown in Table 1.

\section{Variables Which Are Internal to RESP}

TRC, TIC, BRC, BIC, TRS, TIS, BRS, BIS-refer to the parts of two complex fractions representing the combined transfer function for a continuous and sampled system.

For the continuous system

$$
H(j \omega)=\frac{T R C+j T I C}{B R C+j B I C}
$$

for the sampled system

$$
G(j \omega)=\frac{T R S+j T I S}{B R S+j B I S}
$$

CFREQ is a scaling value which keeps the floating-point exponents of the values in $\mathrm{H}(\mathrm{j} \omega)$ from becoming too large. It can be set to any value for which exponent errors do not occur for a particular computer. In general, CFREQ should be equal to the frequency at the center of the range of interest. 
RESP and RESPS work together as a unit and should not require any alterations. The flow of the program operation is as explained in the following steps. (1) RESP is called by a main program, and the specifications for a particular filter system are passed to it. The frequency at which the response is to be computed and the sampling interval are also passed. (2) The eight components of two complex fractions are initialized so both fractions are equal to 1. (3) RESPS is called once for each specified component in order to multiply the complex fractions by the appropriate terms, as illustrated in Table 1. (4) The amplitude and phase response of the continuous and sampled systems are computed as in Eqs. 4-11. (5) Control is returned to the calling program.

The amplitude responses reflect variable gains in the filters, and it is handy to express them in decibels relative to the filter's zero-frequency response. If $X_{0}$ is the response of a particular filter system when $\omega=0$, then

$$
X_{p} \text { in } d B=20 * \log _{10}\left(\frac{X_{p}}{X_{0}}\right)
$$

This will not work for a single zero at 0 , because in this case $\mathrm{X}_{0}=0$.

Phase responses are computed only in the range of $-\pi$ to $\pi$ radians. In interpreting phases, it must be remembered that they are additive and continuous. When the program gives phase values which seem to go from 0 to $\pi$ and then from $-\pi$ to 0 , the values probably represent the range 0 to $2 \pi$. It is convenient to divide phase response by $\pi$ so that phase values will range between -1 and 1 .

The sample program MRESP is an on-line program for finding the response of a given system. $\operatorname{READ}\left(1,{ }^{*}\right)$ means read from the CRT console in free field (needing no format). WRITE $(6, n)$ means write on the Teletype by Format $\mathrm{n}$. Example $\mathrm{D} 1$ shows the response of a single pole at $-1,000 \mathrm{~Hz}$. Note that the pole value is entered as 1000. Specification frequencies are entered with the signs they need to substitute into the equations and not the signs they would have as roots. The output of Example D1 is of the same form as is plotted in Fig. 6.

Example D2 shows the computation of the response characteristics of a much more complex system. This is the linear part of the initial basilar membrane model of Lovell, Carterette, and Nagel (1972).

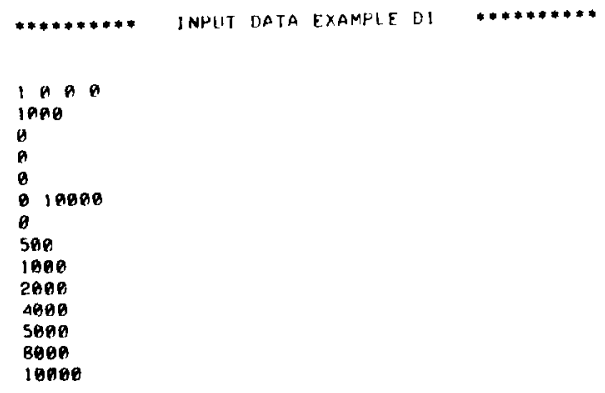

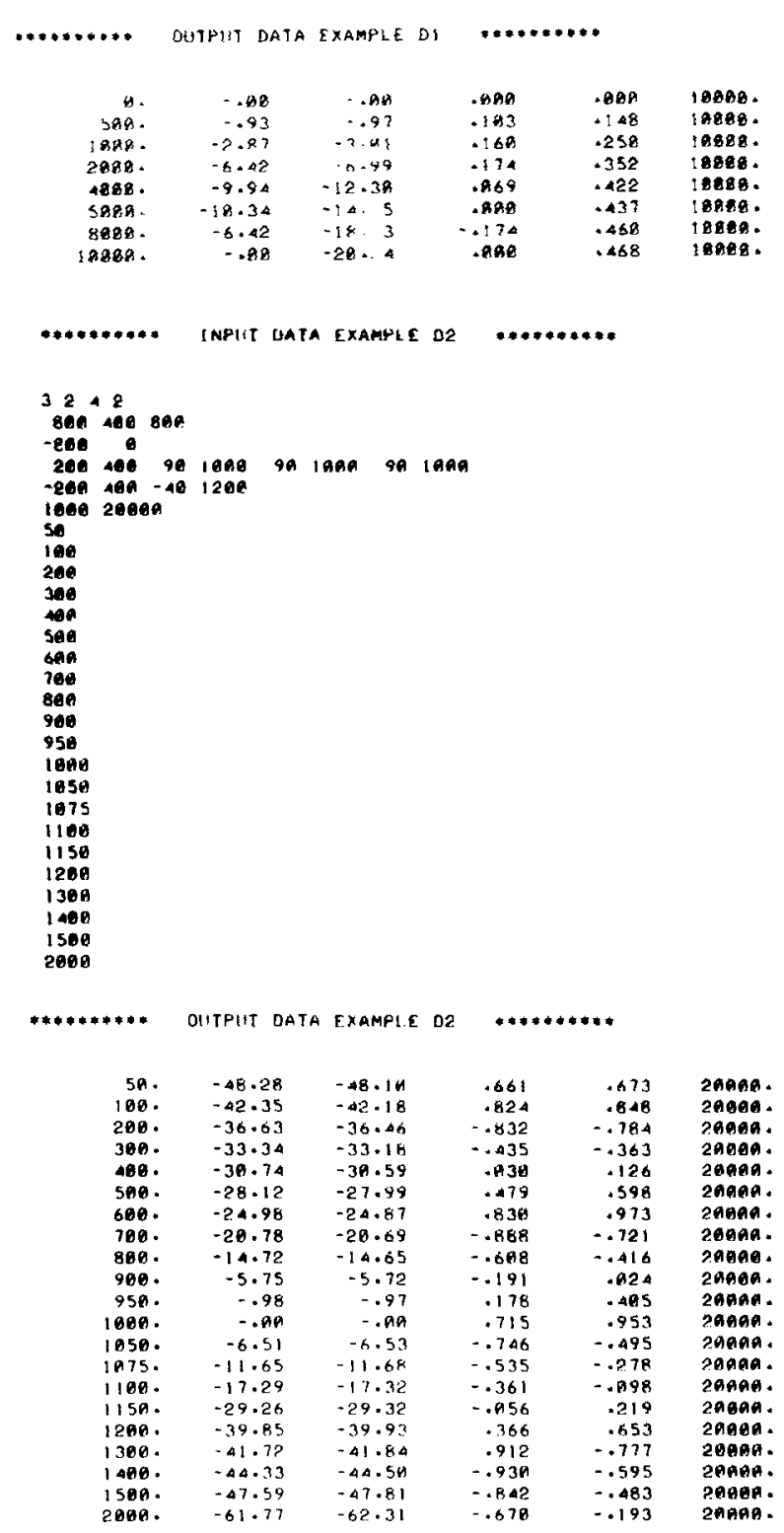

\section{DIGITAL FILTER IMPLEMENTATION}

Once a filter has been designed, it can be implemented by using the same specifications with Programs FILT and FILTS.

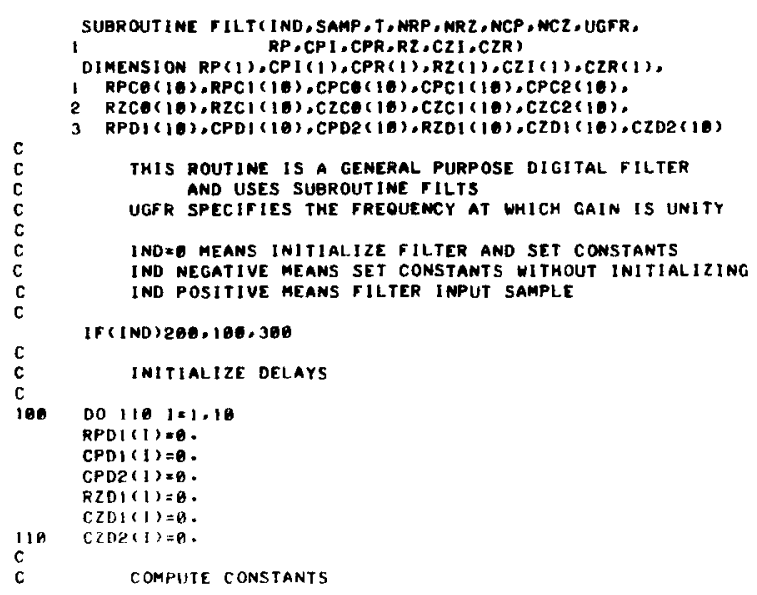




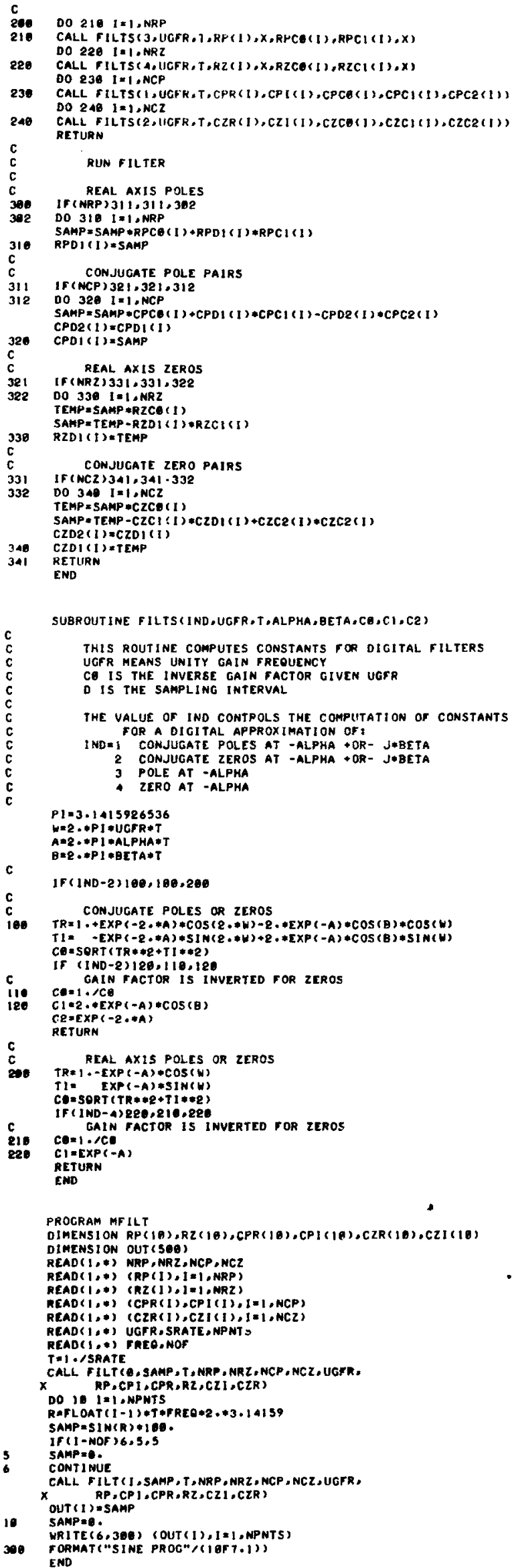

A main program must be supplied by the user to specify a particular filter and supply an input waveform. MFILT is a sample main program for illustration. In addition to the variable specified in the description of RESP, three new variables are in the call statement of FILT:

IND determines the function of FILT as explained in the program comments. Since constants can be changed without reinitializing, the filter characteristics can be varied during processing.

SAMP is the input sample and the output sample.

UGFR is the frequency at which the amplitude response of the system equals unity. This can be any value and would generally be the frequency of greatest interest.

\section{Variables Internal to FILT}

The four arrays ending in $\mathrm{CO}$ refer to the inverse gain factors for the four kinds of components.

The eight arrays ending in $\mathrm{C} 1$ and $\mathrm{C} 2$ are the constants in the difference equations, as in Fig. 4.

The six arrays ending in D1 and D2 are the delay components, as shown in Fig. 4.

FILTS is a necessary subroutine to FILT, but it is written so as to be useful in setting the constants for other digital filter programs.

IND determines the function of FILTS and is described in program comments.

ALPHA and BETA specify a system component.

$\mathrm{C} 0$ is the inverse gain factor necessary to make the gain at UGFR equal to 1 . The method of computation is the same as in the program RESP.

$\mathrm{C} 1$ and $\mathrm{C} 2$ are constants used in the difference equations shown in Fig. 4. For single poles or zeroes, BETA and $\mathrm{C} 2$ are not used.

FILT and FILTS together form a programmable general-purpose digital filter. The flow of the program operation is as follows: (1) FILT is called by a main program with filter specification and IND $=0$. This causes the constants to be computed and the system to be initialized at a state of rest. Control returns to the main program. If IND is negative, the system is not initialized at rest. (2) FILT is called again with IND positive and an input waveform sample. (3) Program control goes from Statement 300 to Statement 340, and the input sample is passed progressively through one difference equation for each component in the digital filter. At the end of this sequence, the input sample has been transformed into the output sample. (4) Control is returned to the main program and can be looped back to Step 1 or Step 2.

It is easy to get an impulse function for a filter system by giving it one nonzero sample followed by zeroes.

Figure 8 shows two examples of using the program MFILT to realize a digital filter. Two cycles of a $1,000-\mathrm{Hz}$ sine wave are input to digital filters at a rate of $50 \mathrm{~K}$ samples per second. In Example U1, the filter is a single pole at -1000 , and in Example U2, the filter is a single zero at -1000 . In both cases, the filters are set for 
Fig. 8. The dashed line represents two into a digital filter. The solid line represents the associated output from a digital filter comprised of one real pole with $\alpha=$ $-1,000 \mathrm{~Hz}$ (see Example U1 for the program filter specification). The response of this system to a $1,000-\mathrm{Hz}$ sinusoid should be that the amplitude is down by $3 \mathrm{~dB}$ and the phase lags by $\pi / 4$. The dotted line represents the output from a digital filter comprised of one real zero with $\alpha=$ $-1,000 \mathrm{~Hz}$ (see Example U2 for the program filter specification). The response of this system to a $1,000-\mathrm{Hz}$ sinusoid should be that the amplitude is greater by $3 \mathrm{~dB}$ and the phase leads by $\pi / 4$. cycles of a $100-\mathrm{Hz}$ sine wave that is input

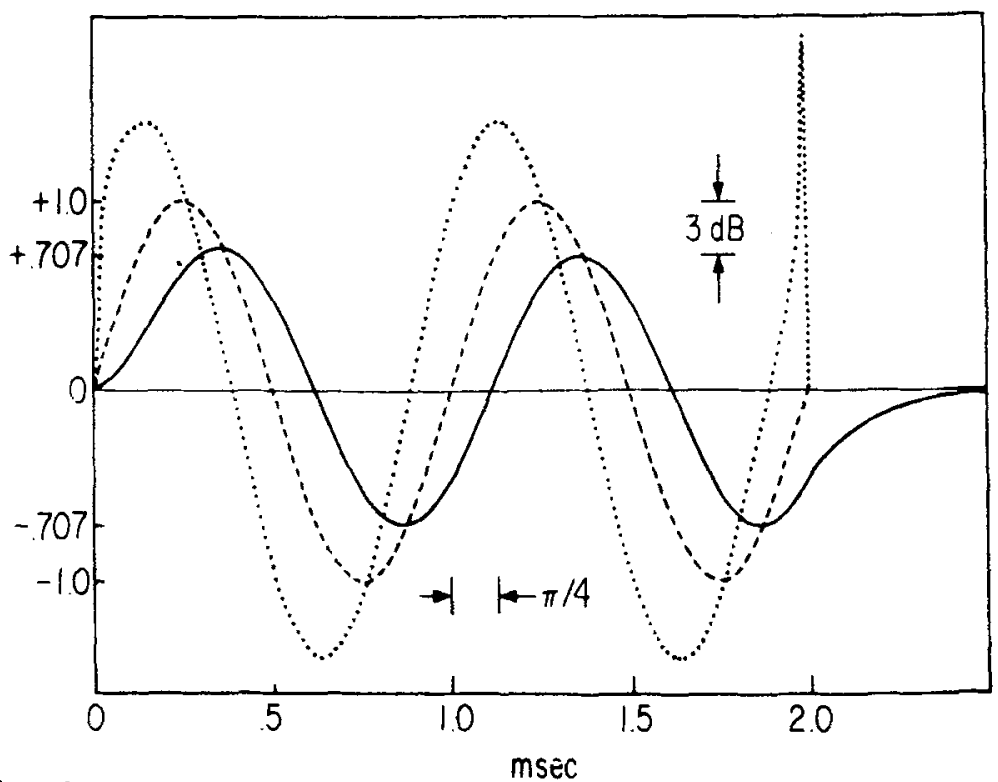

unity gain at zero frequency. Thus, the pole reduces the amplitude of the sine-wave input and produces phase lag. The zero increases the amplitude of the sine input and produces phase lead.
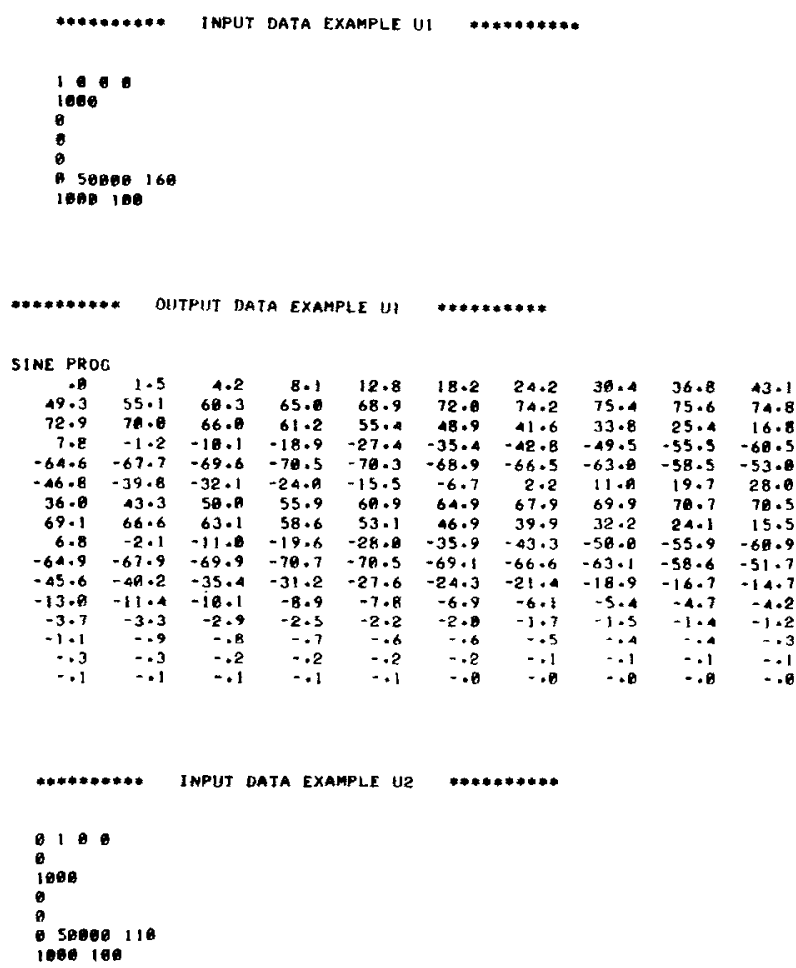

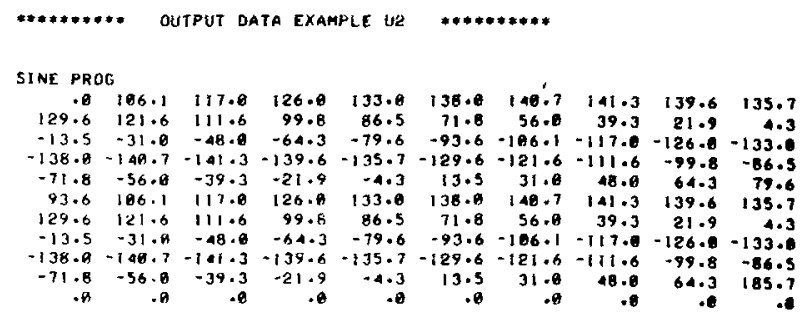

\section{REFERENCES}

Avenhus, E. On the design of digital filters with coefficients of limited word length. Institute of Electrical \& Electronics Engineers, Transactions on Audio \& Electroacoustics, 1972, $A U-20,206-212$.

Churchill, R. V. Operational mathematics. New York: McGraw-Hill, 1958.

Flanagan, J. L. Speech analysis synthesis and perception. (2nd ed.) New York: Academic Press, 1972.

Gold, B., \& Rader, C. M. Digital processing of signals. New York: McGraw-Hill, 1969.

Hamming. R. W. Introduction to applied numerical analysis. New York: McGraw-Hill, 1971.

Jury, E. I. Theory and application of the $z$-transform method. New York: Wiley, 1964.

Kaiser, J. F. Digital filters. In F. F. Kuo and J. F, Kaiser (Eds.), System analysis by digital computer. New York: Wiley, 1966.

Lovell, J. D., Carterette, E. C.. \& Nazel, D. C. Digital simulation model of the basilar membrane. Journal of the Acoustical Society of America, 1972, 51,93.

Oppenheim, A. V. (Ed.) Papers on signal processing. Cambridge: M.I.T. Press, 1969.

Schafer, R. W. A survey of digital speech processing techniques. Institute of Electrical \& Electronics Engineers, Transactions 'on Audio \& Electroacoustics, 1972, AU-20, 28-35.

Weaver, C. S., Mantey, P. E., Lawrence, R. W., \& Cole, C. A. Digital spectrum analyzers. Systems Techniques Laboratory, Stanford, California, 1966, Technical Reports 1809-1 and $1810-1,93 \mathrm{pp}$.

Zwislocki, J. J. Electrical model of the middle eax. Journal of the Acoustical Society of America, 1959, 31, 841 .

(Received for publication October 9, 1972;

revision received November 6,1972 .) 